\title{
SkinHaptics: Ultrasound Focused in the Hand Creates Tactile Sensations
}

\author{
Daniel Spelmezan $^{1} \quad$ Rafael Morales González ${ }^{2} \quad$ Sriram Subramanian $^{3}$
}

\begin{abstract}
Recent developments in on-body interfaces have extended the interaction space of physical devices to the skin of our hands. While these interfaces can easily project graphical elements on the bare hand, they cannot give tactile feedback. Here we present a technology that could help to expand the output capability of on-body interfaces to provide tactile feedback without restricting the skin as an interaction surface. SkinHaptics works by focusing ultrasound in the hand using a phased array of ultrasound transmitters and the acoustic timereversal signal processing technique. We present experimental results that show that this device can steer and focus ultrasound on the skin through the hand, which provides the basis for the envisioned technology. We then present results of a study that show that the focused energy can create sensations that are perceived under the skin and in the hand. We demonstrate the potential of SkinHaptics and discuss how our proof-of-concept device can be scaled beyond the prototype.
\end{abstract}

\section{INTRODUCTION}

Our hands and forearms are a natural interaction platform for on-body interfaces that use the skin as an input and output surface. Unlike visual output, which can be displayed with pico- and laser-projectors [1], [2], [3], no practical solution exists to give tactile feedback without placing actuators on the skin, although tactile cues are as important as visual cues for precise pointing (e.g., for eyes-free interaction [4]). Recently, a few technologies have emerged that can give tactile feedback from a distance with air vortices [5], with focused airborne ultrasound [6], or with indirect laser radiation [7]. However, they are not wearable and have a limited haptic design space. Air vortices and airborne ultrasound create low-resolution sensations that resemble a puff of air, whereas laser requires instrumenting the skin with a light-absorbing medium to elicit a sensation similar to a tap.

Here, we propose SkinHaptics, a new type of haptic feedback that is created by focusing ultrasound through the hand using a phased array of ultrasound transmitters (see Fig. 1). The device can be worn on the side of the hand opposite to the interaction surface requested by an on-body interface and does not interfere with interactive elements that the interface could provide (e.g., a menu projected on the skin [1], [2]). In our current implementation the focused acoustic energy can create sensations that seem to be located in the hand. We ultimately envision to be able to create sensations on the skin at the opposite side of the hand.

This work was supported by Nokia Research Centre and the European Research Council (Proof-of-concept - 640749) under the H2020 Programme.

${ }^{1}$ Daniel Spelmezan is with University of Sussex, United Kingdom d.spelmezandsussex.ac.uk

${ }^{2}$ Rafael M. González is with INRIA, Univ. Paris-Sud \& CNRS, France rafael.moralesdinria.fr

${ }^{3}$ Sriram Subramanian is with University of Sussex, United Kingdom sriramesussex.ac.uk

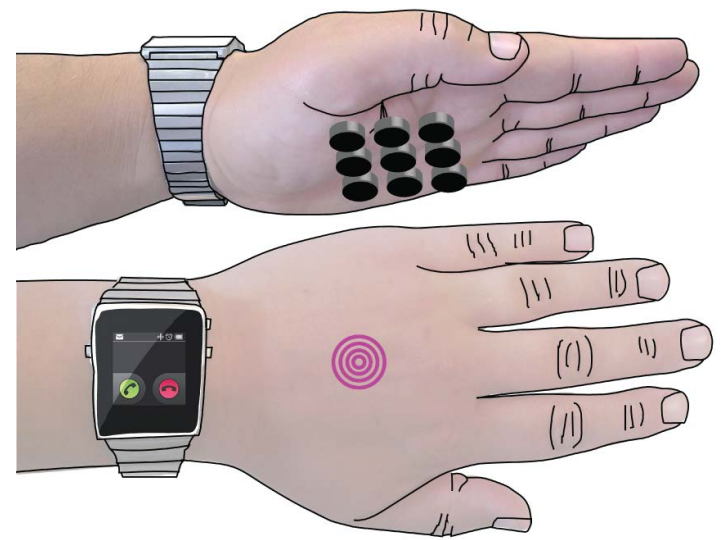

Fig. 1. Ultrasound transmitters emit acoustic energy into the hand. The pressure waves interfere constructively to create tactile feedback in the hand and through the hand (e.g., for smartwatch interactions [3]). Alternatively, the transmitters could be placed at the back of the hand to create tactile feedback at the palm (e.g., for palm-based interfaces [1], [2], [4]).

Our motivation for exploring this technology is grounded in the wide use of ultrasound in medical applications (e.g., for imaging diagnosis and therapy [8], [9]). Moreover, in medical science focused ultrasound has been used as a noninvasive method for diagnosing hearing and neurological disorders, and for creating tactile, temperature and pain sensations in the hand [10]. As a first step to investigate how ultrasound focused in the hand could provide tactile feedback for human-computer interaction, we present a technology that provides the basis for the device we envision and explore the fundamental requirements for creating tactile sensations.

We make the following contributions to demonstrate the feasibility of SkinHaptics: (1) We present the system we developed for focusing ultrasound through the hand using the acoustic time reversal signal processing technique [11], [12]. (2) We conducted two system evaluations using lowintensity, low-frequency ultrasound. In the first informal evaluation we verified that the system can steer and focus ultrasound through the hand. In the second formal evaluation we measured the distribution of acoustic energy at the back of the hand and identified regions with good focusing quality. (3) We conducted a preliminary user study on evoking tactile sensations with focused ultrasound and with unfocused ultrasound. We found that only focused ultrasound created noticeable sensations that were perceived slightly in the hand, which confirmed that SkinHaptics works.

\section{SKInHAPTICS: BACKGROUND AND PRINCIPLE}

Our work is related to haptic feedback devices, focused ultrasound for stimulating neuroreceptor structures for medical diagnosis, and time-reversed acoustics. 


\section{A. Haptic feedback devices}

Research has looked at numerous interfaces for creating tactile sensations (e.g., mechanical vibrations with pin arrays and vibration motors). Our work is similar to electrocutaneous stimulation with which electrical current is sent into the skin from a matrix of electrodes [13]. Common to these interfaces is that they can create sensations only in the skin area that they are in contact with, and not through the hand.

Recently, non-contact haptic feedback devices that can stimulate the skin from a distance have received attention in human-computer interaction (e.g., those that generate and shoot air-vortices [5]). Most similar to our work are phased arrays of ultrasound transmitters used to create a focal area of high sound pressure level in mid-air [6]. Unlike airborne ultrasound, we envision using the body as propagation medium for the acoustic energy to create the focal point in the hand. However, the techniques that generate a focal point in midair can work only in a homogeneous medium. To focus ultrasound through an inhomogeneous medium such as the hand, in which the spatial and temporal shape of the signal is distorted, a different technique is needed (e.g., a phase conjugate mirror or an acoustic time reversal mirror [12]).

\section{B. Ultrasound stimulation of neuroreceptor structures}

One of the most intriguing methods for creating tactile sensations in the hand involves focused ultrasound [10], [14]. Gavrilov et al. investigated this method as a noninvasive tool for diagnosing diseases that affect the nervous system (e.g., the hearing or the skin). They directed ultrasound pulses to the hand using a pre-focused cylindrical transducer $(0.48-$ 2.67 megahertz) and short stimuli (1-100 milliseconds) with intensities up to several thousands watts per square centimeter [15]. With increasing intensity the focused beam created first tactile, then temperature, and lastly pain sensations. The tactile sensations resembled a slight stroke or push and were reproducible, unless the focal region of the beam was entirely in the soft tissues under the skin, in which case only sensations forerunning pain or pain were caused. Relevant to our work is that the tactile sensations reappeared when the focal region was moved through the soft tissues onto the skin layer at the other side of the hand (e.g., at the tissues between the thumb and the forefinger), albeit at higher intensities due to the attenuation of energy in the tissue.

The main cause of the tactile sensations is the radiation force in the focal region, which displaces the medium in one direction, thereby stimulating the mechanoreceptors [14], [16]. Gavrilov et al. found that the displacement amplitude was approximately 0.1 micrometer for all threshold intensities and independent of the frequency. However, biological effects (e.g., heating) and the direct action of ultrasound vibrations on nerve fibres could be relevant as well [10].

\section{Time-reversed acoustics}

We use acoustic time reversal to focus ultrasound through the hand. Unlike time-delay focusing techniques, which can focus sound in a homogeneous medium, time reversal

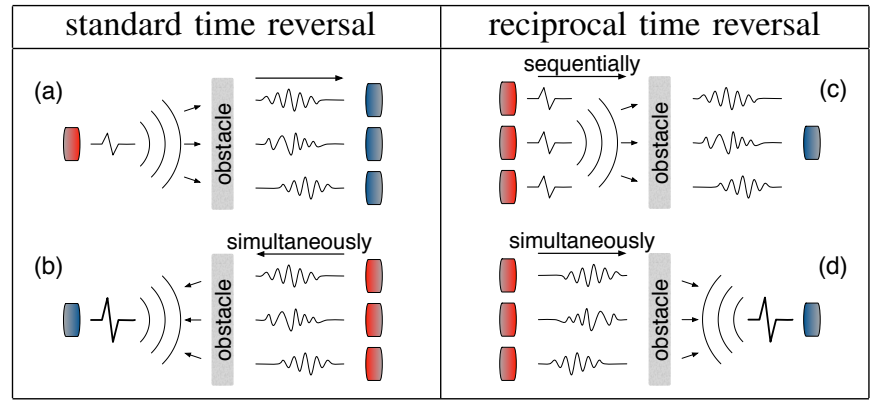

Fig. 2. The acoustic time reversal mirror implemented with standard (a-b) and with reciprocal time reversal (c-d) to create the same focal point (b,d). The transmitters are shown in red and the receivers in blue.

signal processing can focus sound through an inhomogeneous medium (e.g., tissue) [12]. Its principle is the spatial reciprocity of waves. The symmetry with which sound waves travel between two points when either point is used as a source to emit an impulse allows capturing a diverging wave front and retransmitting the signals to create a converging wave front that propagates back to the source location (an acoustic time reversal mirror). This property has enabled numerous applications (e.g., estimating the origin of earthquakes [17], localizing and destroying kidney stones [17], sensing the location of finger taps [18], [19], and providing tactile feedback on touch panels [18], [20]).

1) Standard time reversal: Fig. 2 illustrates the principle of an acoustic time reversal mirror. In the common implementation a signal source (an active transmitter or a passive reflector) generates an impulse, which propagates as a wave field through the medium (see Fig. 2-a). Obstacles in the sound propagation path (sound speed inhomogeneities) distort the spatial and temporal shape of the wavefronts through multiple reflections, scattering, and diffraction. The distorted wave field is recorded at several receiver locations in the medium. When the receivers simultaneously retransmit the signals in reversed chronological order, the waves propagate back and refocus at the location of the source as an impulse similar to the original impulse (see Fig. 2-b). The mirror operation (time-reversing the signals and back-propagation through the medium) corrects the distorted wave field.

2) Reciprocal time reversal: The spatial reciprocity of wave propagation allows interchanging the transmitters and the receivers to obtain the identical focused signal as in standard time reversal [17], thereby focusing the wave field at the location of the receiver instead of the source. In this implementation the transmitters sequentially emit the same impulse from different locations, and the receiver records the respective impulse responses (see Fig. 2-c). When the transmitters simultaneously retransmit their respective impulse responses in reversed chronological order the reversed signals focus as an impulse at the location of the receiver (see Fig. 2-d). We used this approach to implement SkinHaptics, which allowed us to simplify the hardware and to build separate circuits for the transmitters and for the receiver.

3) Constructive interference and one-bit quantization: The time reversal mirror retransmits the signals with their respective phases and amplitudes to recreate the impulse. The 


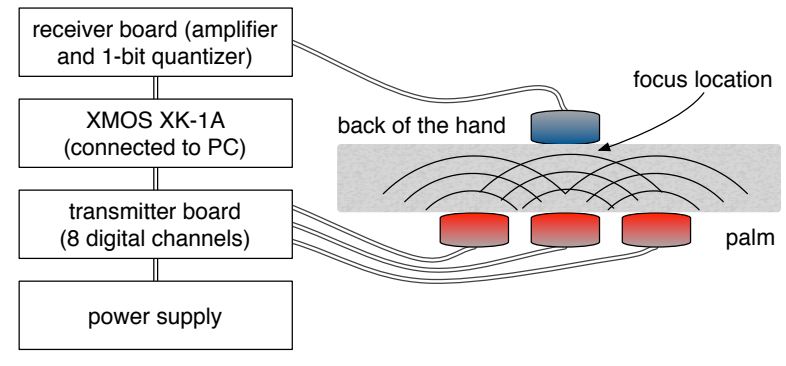

Fig. 3. The setup used for focusing ultrasound from the transmitters (red) to the receiver location (blue) with reciprocal time-reversal.

amplitude at the focus location is the sum of the amplitudes of the individual waves [19]. To perform time reversal only information on the instantaneous phase is needed because the phase difference between the waves determines the interference pattern at any location in the medium. This relationship allows reducing the resolution with which the wave amplitude is recorded to one bit. With one-bit time reversal only the sign $( \pm)$ of the amplitude is recorded and used during the retransmission to set the signal amplitude $( \pm 1)$, which has been shown to improve the signal-to-noise ratio and to amplify the time-reversed impulse [21], [22].

4) The size of the focal point: The acoustic time-reversal mirror has a diffraction-limited focusing resolution like an optical lens, which depends foremost on the ultrasound wavelength $(\lambda)$ [11], [12]. Diffraction effects scatter the waves and limit the resolution to the theoretical minimum of $\lambda / 2$ [19]. The resolution could be lower as the array captures and retransmits only a part of the acoustic field, which causes an information loss at the focus location. To accurately reverse the signals and to avoid grating lobes (secondary lobes of high intensity) during the retransmission, the optimal size and pitch of the transducers is $\lambda / 2$ [11], [12].

We used 40 kilohertz for SkinHaptics. We based our choice of frequency on recommendations from Gavrilov et al. who suggested using low frequencies to reduce the intensity needed to create tactile sensations and to minimize the energy loss due to attenuation in tissue [10]. While our frequency is one magnitude smaller than the lowest frequency (480 kilohertz) used in [10], we expected to affect a large area of the hand and to be able to create tactile sensations at low-intensities. We estimated the spread of energy in the focal region to have a minimum diameter of 2 centimeters and possibly to extend through the hand $\left(\lambda=c / f=4\right.$ centimeters, $c_{s k i n} \approx 1600$ meters per second [23], $c_{\text {soft tissue }} \approx 1540$ meters per second [24]).

\section{THE HARDWARE FOR SKINHAPTICS}

Our prototype consists of an array of transmitters at one side of the hand and one receiver that is temporarily placed at the other side of the hand to set the location of the focal point (see Fig. 3). This setup has some similarities to the hardware with which ultrasound (35-50 kilohertz) was emitted into the forearm and sensed with one receiver to detect touch gestures on the skin [25]. Unlike the approach used in [25], we focus the energy through the hand at the location of the receiver.

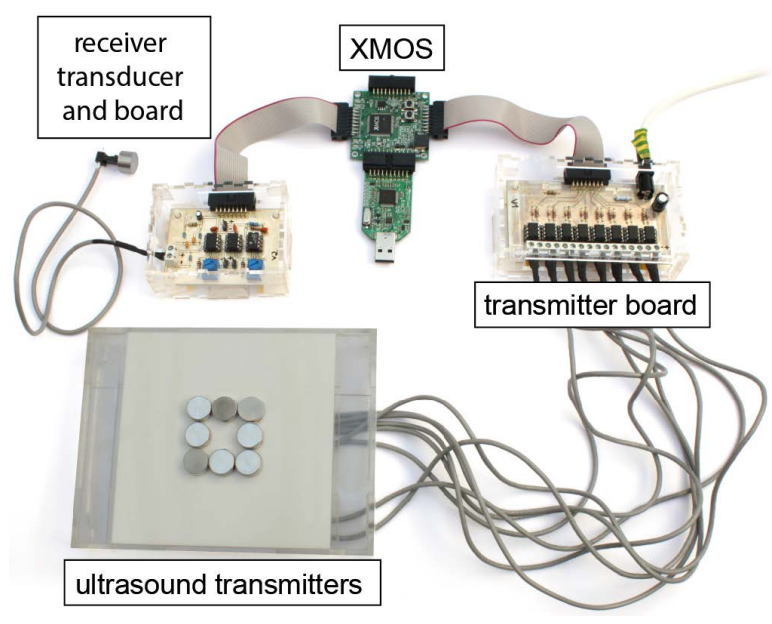

Fig. 4. The SkinHaptics prototype.

\section{A. Hardware design and system setup}

To focus ultrasound on the skin through the hand we implemented reciprocal one-bit time reversal using eight transducers connected to a transmitter circuit board, one transducer connected to a receiver circuit board, and one XMOS XK-1A micro-controller (see Fig. 3 and Fig. 4).

1) Ultrasound transducers: The transmitters and the receiver are off-the-shelf 40 kilohertz transducers (Multicomp MCUSD14A40S09RS). The piezo disc (diameter of 7 millimeters) is built inside an aluminum housing (diameter of 14 millimeters) and has a low directivity ( $110 \times 50$ degrees $)$. The housing does not have an acoustic matching layer for maximizing the energy transmission to the skin. However, we found that the energy spread widely through the hand and allowed us to receive the signals from all transmitters.

2) Transmitter and receiver circuit boards: The circuit boards are connected to the digital pins of the XMOS. The transmitter board controls eight switching MOSFETs (TC4428A, maximum 18 Volts) driven by square waves. The receiver board amplifies and quantizes the received analog signals using two op-amps (LF411) and one voltage comparator (LM393) for input to the XMOS. The comparator converts the analog signal to one-bit resolution, allowing us to perform time-reversal using only digital I/O (see Fig. 5).

3) Software implementation (calibration and focusing):

Table I lists the steps we implemented to perform reciprocal time reversal. (1) To calibrate the system we activate the transmitters separately and record the impulse response at the intended focus location at the back of the hand. Each transmitter emits an impulse of ten cycles at 40 kilohertz, while the XMOS samples the quantized signal at 10 megahertz and saves the bits $(0 / 1)$ in a buffer of 500 microseconds length (see Fig. 5). (2) The buffer is subsequently merged with the time-reversed buffer, which represents the time-correlated impulse responses from all transmitters. (3) The receiver is removed from the skin as it is needed only to set the focus location. (4) To create the focal point all transmitters are simultaneously activated using the time-reversed buffer. 


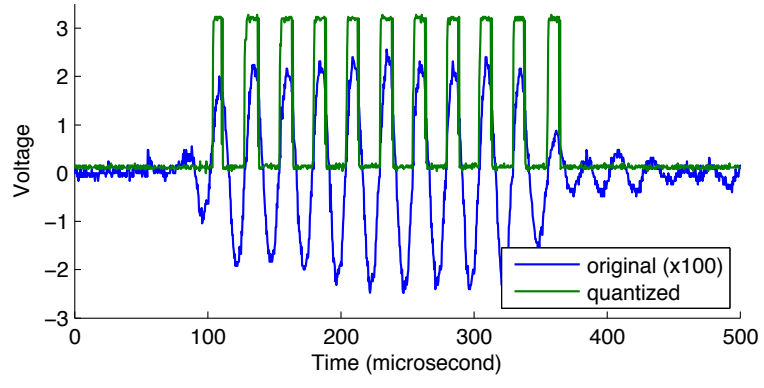

Fig. 5. One-bit quantization of analog signals. This impulse response was sensed at the back of the hand when one transmitter was pulsed to emit the calibration signal at the center of the palm. The quantized signal represents high (1) and low (0) at TTL logic level.

4) Setup for experiments: We placed the palm on the transmitters and the receiver at the back of the hand (see Fig. 1 and Fig. 3). We chose this configuration to ensure that the large transducer housing rested flat on the skin without introducing air gaps that would reflect ultrasound, which was difficult to achieve when the transmitters were placed on the extrinsic muscles at the back of the hand (smaller transducers would be appropriate). Note that our configuration does not introduce any limitations for evaluating the SkinHaptics prototype, as focusing can be performed equally well in both directions due to the principle of spatial reciprocity.

\section{ULTRASOUND SAFETY}

Ultrasound can interact with biological tissue through thermal and mechanical mechanisms and can cause effects such as heating and cavitation at high intensities [9], [24], [26]. These effects can be harmful. However, in controlled conditions they form the basis for therapeutic applications (e.g., in watts per square centimeter: bone fracture healing 0.030 , physiotherapy 1 , and 1000 for selective heating and destroying of tissue with high-intensity focused ultrasound [8], [26]). Gavrilov et al. used high intensities with brief exposure durations (thousands of watts, 1-100 milliseconds) to create tactile sensations in the hand for medical research [10]. Presumably, those intensities did not cause morphological changes, nor injure, nor destroy the tissues [10], [27].

1) Ultrasound parameters for creating tactile sensations: In Gavrilov et al.'s experiment [15] the intensity needed to cause tactile sensations indicated a relationship between the stimulus location and the ultrasound parameters, which was attributed to the receptor density in the respective areas [10], [14], [27]. The highest intensity was needed at the forearm. The lowest intensities were needed at the finger and palm ( 8 and 16 watts per square centimeter, 1 millisecond, 0.48 megahertz) [27]. A lower intensity was needed for the lower frequencies, for which the diameter of the focal region was larger and the energy attenuation lower compared to the highest frequency [10]. Although no clear link was found between the exposure duration and the threshold intensity, thermal effects caused by the energy attenuation in tissue could be relevant for long stimuli and high frequencies [27].

2) Ultrasound intensity for SkinHaptics: Nowadays, internationally accepted guidelines for the safe use of ultrasound
TABLE I

THE CALIBRATION AND FOCUSING PROCEDURE.

Calibration (for each transmitter separately, cf. Fig. 2-c)

1. Emit 10 cycles and record the impulse response.

2. Adjust the time-reversed buffer.

Focusing (with all transmitters simultaneously, cf. Fig. 2-d)

3. Remove the receiver from the hand.

4. Transmit the time-reversed buffer.

regulate the application of high intensities. Experiments conducted with human participants outside a medical research laboratory are subject to recommendations for diagnostic ultrasound with an intensity limit of 0.72 watt per square centimeter spatial-peak temporal-average (i.e., time-averaged over the pulse-repetition period) [8], [28]. This limit is considered to be safe. It is, however, lower than the threshold intensity of tactile sensations in the hand [14], [27].

We estimated the acoustic power emitted into the hand from the electrical power delivered to the system [8]. The output of one transmitter is 0.32 watt at 15 volts (cf. 0.2 watt [25]). The spatial and temporal average intensity at the skin of the palm is approximately 0.013 watt per square centimeter when we consider the area of the piezo disc, the impedance mismatch at the transmitter-skin interface $(31 \%$ transmission coefficient, $Z_{A l}=17$ [29], $Z_{\text {skin }}=1.6$ [23], $Z$ in $10^{6} \mathrm{kgm}^{-2} \mathrm{~s}^{-1}$ ), and pulsed ultrasound with $5 \%$ duty cycle. To estimate the intensity at the focal region in the hand we need to consider the focusing quality of the time reversal mirror and the attenuation of energy in the tissues. For a conservative estimate of the focused intensity from eight transmitters we can assume that the maximum is in the order of 0.1 watt per square centimeter.

\section{Evaluation}

Our goal was to assess if we can focus ultrasound through the hand and if the focused energy can elicit a sensation. We started with the technical evaluation to verify that acoustic time reversal can direct ultrasound from the palm towards a specific location at the back of the hand. We then conducted a user study about the energy distribution at the focus location. Finally, we piloted an experiment on evoking sensations. The studies were approved by the university's research ethics committee to ensure the safety of the participants.

\section{A. Technical evaluation of focusing ultrasound}

This initial informal self-test verified that our system could transmit and focus ultrasound through the hand. Our main interest was the amplitude of the time-reversed signals. We expected to observe a higher amplitude around the intended focus location than at adjacent locations, which would confirm that the system functioned as intended. The variations in the signal level would indicate the spread of energy and the size of the focal region, which we estimated to have a minimum diameter of 2 centimeters (see section II-C.4). In addition, we logged the signals created when all transmitters were simultaneously pulsed to compare the distribution of energy in the hand when the waves interfered randomly (unfocused) and when they were time-reversed (focused). 


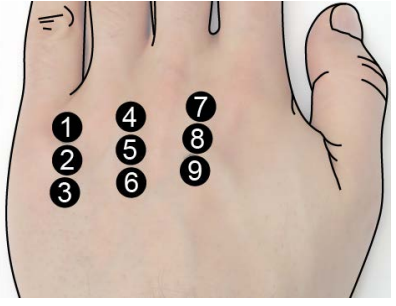

(a)

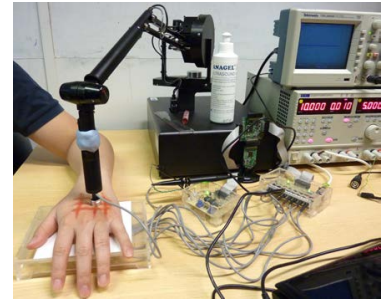

(b)
Fig. 6. The setup used for measuring the time-reversed signals.

1) Participant: We obtained data measurements from the left hand of one of the developers of the system. We used his hand because we did not have to expect a subjective bias when evaluating the functioning of the system.

2) Setup and Procedure: We chose the interosseous metacarpal spaces $(4,3,2)$ between the knuckles to set the focus locations and sampled data towards the middle of the hand (see Fig. 6-a). The hand structure in this area allows focusing between the metacarpal bones where more energy can propagate through the tissues. Nine focus and sampling locations were marked on a grid $(3 \times 3)$. A thin layer of ultrasound gel was applied on both sides of the hand. The palm was placed on the array, and the upper row of transmitters was aligned to the position under the knuckles.

We attached the receiver transducer to the stylus of a Phantom Premium device to ensure a constant pressure of 100 gram during the calibration phase and when logging data (see Fig. 6-b). For each focus location we calibrated the system and re-emitted the time-reversed buffer (see Table I) with 5 milliseconds pulse repetition period and 10\% duty cycle (approximately 38 milliwatts per square centimeter temporal average intensity at the piezo disc at 10 volts). For each sampling location we logged with an oscilloscope one instance of the original analog waveform of the time-reversed signal (1 millisecond at 2.5 megahertz). Subsequently, we simultaneously activated all transmitters with ten cycles to $\log$ the unfocused signals. We recorded $2 \times 9 \times 9=162$ data set. Capturing all sets lasted approximately 90 minutes.

3) Findings: Fig. 7 shows the areas on the hand affected by ultrasound. The unfocused waves have the same energy distribution in all sets (see Fig. 7-a). The unfocused power is maximal at location 7 (0.0073 square volts) and decreases towards the thicker, middle part of the hand, which could be related to the higher energy attenuation towards the carpus.

The focused waves show distinct patterns of energy distributions (see Fig. 7-b). In general, the power is maximal at the focus location and in proximity to this area. Distinct foci emerge when the distance between them is at least in the order of 2-3 centimeters. The focused power is maximal at location 7 (0.0178 square volts) and tends to decrease for foci towards the carpus (e.g., locations 3, 6 and 9). We also observe a larger spread of energy at focus location 5 and a vertical spread for the foci in the 2nd metacarpal space, which could be caused by stronger interaction effects of ultrasound with the tissues and the boundary to bones.

The difference between the maximum of the focused and



(a)

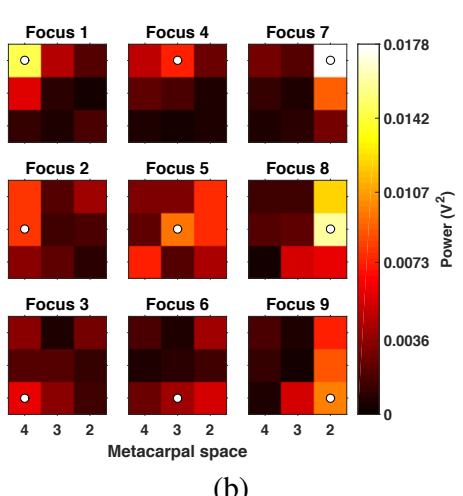

(b)
Fig. 7. Absolute power of (a) unfocused and (b) focused ultrasound pulses. The circles show the focus locations used to calibrate the system.

unfocused power $(2.4 \times)$ and the energy distribution around the intended focus locations confirms that our system can steer and focus ultrasound in a local area on the hand.

\section{B. User study on the distribution of energy at the focus}

We conducted a formal evaluation to verify if our findings were reproducible. We aimed at finding regions with maximal energy and with pronounced boundaries, which could be used for creating localized focal points on the hand.

1) Participants: Eight volunteers (1 woman) participated, age 23-32 years old (average 29.9, median 31).

2) Setup and Procedure: We first informed the participants about the system and the measurements, and asked them to sign a consent form. We then followed the procedure described in our self-test (see section V-A.2 and Fig. 6). For each of the nine focus locations we created a focal point with 100 milliseconds pulse repetition period and $0.5 \%$ duty cycle ( 2 milliwatts per square centimeter temporal average intensity at the piezo disc at 10 volts) and logged for each sampling location the analog waveform of the time-reversed signal. We recorded nine data sets with nine samples per set for each participant. The experiment lasted one hour.

3) Findings: We calculated the power of the signals and normalized the data set belonging to a focus location to the maximum value in this set. Fig. 8-a illustrates the directivity pattern of the time reversal mirror around the intended focus locations as normalized average power. The power is maximal at the calibration point and tends to decrease with increasing distance from this location. The normalized standard deviation among all participants (see Fig. 8-b) indicates some variation in the peak power, which can be attributed to the different sizes of the participants' hands. However, the variation quickly decreases at the adjacent locations and is minimal at the distant locations. This spatial distribution resembles the patterns observed in our self-test (see Fig. 7-b) and confirms that the energy is concentrated in proximity to the focus location specified on the hand.

The spread varies with the focus location. The foci 7 , 8 and 9 spread vertically and have a notable boundary to the left. The other foci are more diffuse. They extend in horizontal and in vertical direction with less pronounced boundaries to the adjacent metacarpal space. These distinct 


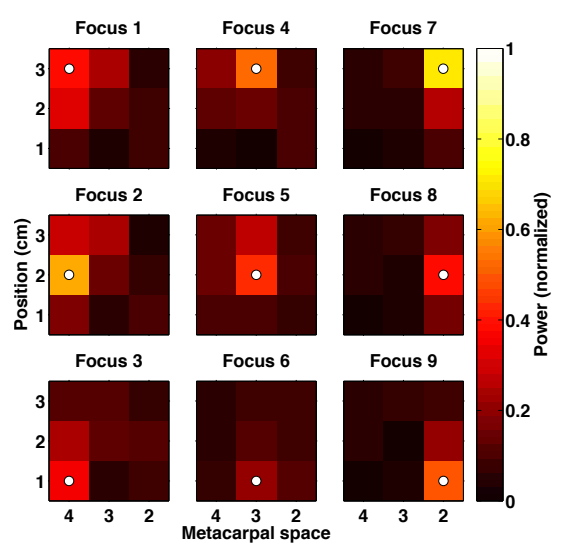

(a)



(b)



(c)

Fig. 8. (a) Normalized average power and (b) normalized standard deviation. The data was normalized for each participant and focus location. (c) Absolute average power measured among the participants' hands. The circles show the focus locations used to calibrate the system.

patterns could be caused by the hand structure and the width of the metacarpal bones (e.g., the average distance between the foci 1 and 4 was 17.8 millimeters and 21.5 between the foci 4 and 7). The steep decrease in power towards the distant locations suggests that the focal region spreads in the order of 2-3 centimeters, which is most prominent for foci at the perimeter and which could be related to the wavelength of ultrasound in the soft tissues of the hand (see section II-C.4).

The absolute average power confirms the peak at the intended focus location (see Fig. 8-c). However, the foci have different energy characteristics. The power varies across the hand and is maximal at the perimeter, foci 2 and 7 , and minimal in the middle of the hand, foci 5 and 6 . The attenuation of energy can be related to the hand structure with its increasing thickness and its decreasing distance between the metacarpal bones towards the carpus. Variations in the hand thickness and width, and the slightly different alignment of the transmitter array under the palm, could also explain the differences in the absolute power of the foci.

\section{User study on evoking sensations in the hand}

Our hardware can only deliver ultrasound of significantly lower intensity than the minimum Gavrilov et al. applied to create tactile sensations for medical research (cf. 8 watts per square centimeter [27]). Therefore, we explored in self-tests if we can find evidence of creating sensations when modulating the signal at lower frequencies (e.g., 200 hertz [14]). We also experimented with repeatedly sending the time-reversed signal in series $(1-1,000 \times)$. The series' duration was 0.5 500 milliseconds with 2 seconds pulse repetition period and $0.025-25 \%$ duty cycle at $10-17$ volts (maximum 0.265 watt per square centimeter at the piezo disc).

The series signal created a sensation at the palm and in the hand similar to a brief pulse, which we noticed for longer durations and higher voltages (e.g., 100 milliseconds, $5 \%$ duty cycle, 40 milliwatts per square centimeter at 15 volts). Sometimes the maximum intensity produced a sharp sensation in the hand. Although reproducible, the occurrence and intensity seemed to also depend on the position of the array and on the ultrasound gel, which made the sensation feel stronger. Occasionally, the focus location seemed to affect the area where we perceived the sensation.

Initially, we had doubts if the focused energy caused the sensation. Another explanation could be the vibration of the transmitters' housing. The duration during which the transmitters are active (the pulse series' length and the pulse repetition period) modulates the 40 kilohertz signal down to a low frequency vibration that could result in a "click" sensation similar to the pulsing of a piezo disc at low frequencies [30]. If this was the case, the sensation would also occur when the emitted signals were unfocused.

To investigate if the focused energy caused the sensation we designed an experiment to compare the focused pulse series to an unfocused pulse series. We introduced an unfocused pulse Sim for which we simultaneously activated the transmitters for the duration of the pulse series using the 40 kilohertz square waves emitted during the calibration phase. We varied the use of ultrasound gel to see if a better coupling between the transmitters and the skin would affect the intensity of the sensation. We also varied the calibration point on the hand to see if the focus location would change the location of the sensation. To keep the study short and to reduce the ultrasound exposure for the participants we set the pulse series duration to 100 milliseconds. The experiment followed a $2 \times 4$ within-participant design with two factors:

- CouplingAgent: NoGel and WithGel;

- Stimulus: F1, F7, F9 and Sim.

We focused the stimuli $F 1, F 7$ and $F 9$ at the locations 1 , 7 and 9 (see Fig. 6-a), which we chose because of their high concentration of energy with a narrow spread, and because the distance between those points is maximal (see Fig. 8). For the unfocused pulse Sim we pretended to calibrate the system at one of the three locations (randomly chosen).

1) Participants: Eight men volunteered, age $23-35$ years old (average 30.3, median 31). Five of them had participated in the first study. None of them had previously experienced the sensations caused by the pulse series.

2) Setup and Procedure: We introduced the participants to the hardware and the purpose of the study, explained the task, and asked them to sign a consent form. The participants 


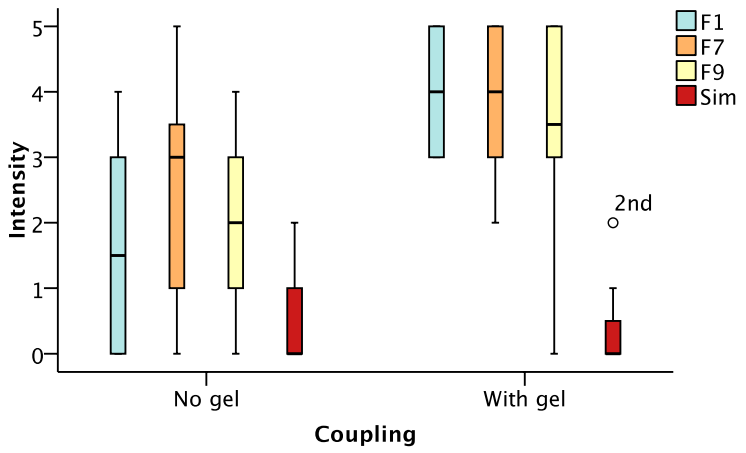

Fig. 9. Likert scale ratings of the perceived intensity of the pulse series $(0=$ no sensation perceived, $1=$ very weak, $2=$ weak, $3=$ neither weak nor strong, $4=$ strong, $5=$ very strong).

sat in a chair and placed their left arm on the table. The experimenter then aligned the palm to the position where the upper row of the transmitters was under the knuckles.

The presentation order of CouplingAgENT and STIMULUS was counterbalanced. Half of the group started without gel at the palm, the other half with gel. All stimuli were presented twice for each condition. For both trials the order of the stimuli was counterbalanced (Latin square).

For each condition we calibrated the system and then removed the receiver from the hand. After five seconds the stimulus (the 100 milliseconds pulse series) was emitted for ten times with 2 seconds pulse repetition period and $5 \%$ duty cycle (40 milliwatts per square centimeter at the piezo disc at 15 volts). The participants rated the intensity of the perceived sensation (6-point Likert scale), described its characteristics in their own words, and marked its location on paper using a sketch of the palm, which we found facilitated interpreting the sensations from this perspective of the hand. For all trials the participants listened to white noise to cancel out audio cues that were created when the transducers were active. The experiment lasted approximately 30 minutes.

3) Findings: Every participant noticed the focused pulse series $(88.5 \%)$ and consistently rated them as more intense with gel than without gel (see Fig. 9). However, the variation in the ratings when no gel was used indicates that the participants perceived the intensity very differently. Their comments included: "weird pulsing", "like a reflex", "I wanted to react [...] to twitch my hand when I felt the strong stimulus for the first time", "electric shock", "not painful", "not scary", "not too bad", "interesting", "the focused pulse on the skin was comfortable [intensity 3]". Four participants noticed the unfocused pulse series Sim $(31.3 \%)$, which resembled a pulse or "wind" in a small area on the skin around the center of the palm, and which was rated to be very weak or weak independent of the gel.

We analyzed the intensity ratings with a full-factorial repeated measures ANOVA after processing the data with the aligned rank transform [31]. We found a significant main effect of CouplingAGEnT $\left(F_{1,7}=12.45, p=.01, r=.79\right)$ and a main effect of STIMULUS $\left(F_{3,21}=27.55, p<.001\right)$, for which a post-hoc t-test with Bonferroni correction showed that the unfocused pulse Sim was of lower intensity than the focused pulses $(p=.011)$. The analysis also showed a significant interaction effect between COUPLINGAGENT and STIMULUS $\left(F_{3,21}=11.0, p<.001\right)$. The focused series were more intense with gel than without gel, but the unfocused series Sim felt weaker with gel than without gel. These results suggest that the gel increased the transmission of ultrasound to the skin and that the focused energy could be the main cause of the experienced sensations.

Fig. 10-b,c shows the most frequent locations and the characteristics of the sensations associated with the focused pulse series. Independent of the gel and the calibration points the sensation was described to have characteristics like "small", "pointlike" or "large", "spread". With gel they were noticed on or in the skin, or deeper in the hand. Without gel they were perceived mostly on or in the skin. For Fl the sensation was located towards the left side of the hand. However, we could not definitely relate the calibration point to a specific location where the sensation was perceived. Four participants described larger sensations that extended on or under the skin towards the fingers, which were sporadic and difficult to reproduce (see Fig. 10-d,e,f,g,h).

These findings illustrate that the focused energy interacted with the hand tissues. However, we were not able to control the location of the sensation. We believe that the sensation was most noticeable at the palm because of its high receptor density. The size of the focal region and possibly energy reflected from the palm could have stimulated several receptors, which created the larger, spread sensations.

The progressive loss of energy due to attenuation in the tissues could have prevented the sensation to occur at the back of the hand. Even so, the low-frequency focus extended in the hand where it was perceived at a significantly lower intensity than reported for high frequencies (see section IV). We asume that higher ultrasound frequencies could help to better localize the sensation, but the energy would attenuate faster and would require higher intensities to propagate through the hand. Clearly, more experiments are needed to study how ultrasound can be used for creating localized tactile sensations in and through the hand, and to ensure the long-term safety of the envisioned technology.

\section{APPLICATION SCENARIOS}

The SkinHaptics technology is worn at one side of the hand and leaves the other side unencumbered for interaction. We believe this interface opens a new design space for haptic sensations in two main application areas. The first involves situations where the user's hand is protected from the environment by an unnatural barrier and deprived from all natural tactile feedback when operating a tool (e.g., spacesuits and protective work gloves). The second is for augmenting on-body interfaces that use the bare hand as an interactive surface (e.g., to signal events or to help the user locate interactive elements during eyes-free interaction).

\section{LIMITATIONS AND FUTURE WORK}

Our findings show that we can steer and focus ultrasound through the hand with an acoustic time reversal mirror 


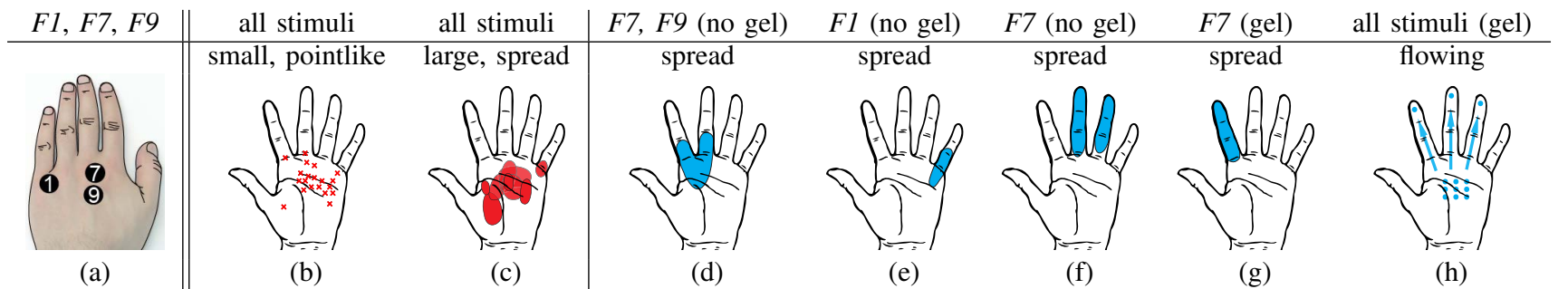

Fig. 10. (a) Focus locations at the back of the hand. (b-h) Characteristics of the sensations and the locations where the focused pulse series was perceived. $(b, c)$ Frequent sensations on the skin, in the skin, or deeper in the hand. $(d-h)$ Infrequent sensations on or under the skin $(2-3 \times)$.

and that the focused energy can cause sensations that are perceived in the skin and deeper in the hand. However, we tested only one specific pulse series for which we did not vary the ultrasound intensity, nor the duration or the transmitter characteristics (e.g., size, directivity and coupling efficiency). Moreover, the frequency affects the size of the focal region and the optimal geometry of the focusing array [9], [11], [12], as well as the attenuation of energy that can change the intensity needed to create tactile sensations by several orders of magnitude [10]. For future work we intend to explore these parameters to better understand the conditions under which to focus the tactile sensations.

Our technology requires tight contact between the skin and transmitters and calibration to set the focus location. Recalibration is needed when the hand or the array is moved. We surmise that a one-time per-user calibration for different hand postures and array positions could be feasible with a transmit-receive array for sensing the posture from the waves reflected in the hand (similar to ultrasound imaging), and emitting the impulse responses that refocus the waves.

We hope that our work demonstrates the possibilities SkinHaptics offers for human-computer interaction and inspires other researchers to explore this technology.

\section{REFERENCES}

[1] C. Harrison, S. Ramamurthy, and S. E. Hudson, "On-body interaction: Armed and dangerous," in Proc. TEI. ACM, 2012, pp. 69-76.

[2] C. Harrison, D. Tan, and D. Morris, "Skinput: Appropriating the body as an input surface," in Proc. CHI. ACM, 2010, pp. 453-462.

[3] G. Laput, R. Xiao, X. A. Chen, S. E. Hudson, and C. Harrison, "Skin buttons: Cheap, small, low-powered and clickable fixed-icon laser projectors," in Proc. ACM UIST. ACM, 2014, pp. 389-394.

[4] S. G. Gustafson, B. Rabe, and P. M. Baudisch, "Understanding palmbased imaginary interfaces: The role of visual and tactile cues when browsing," in Proc. CHI. ACM, 2013, pp. 889-898.

[5] R. Sodhi, I. Poupyrev, M. Glisson, and A. Israr, "Aireal: Interactive tactile experiences in free air," ACM Trans. Graph., vol. 32, no. 4, pp. 134:1-134:10, July 2013.

[6] T. Hoshi, M. Takahashi, T. Iwamoto, and H. Shinoda, "Noncontact tactile display based on radiation pressure of airborne ultrasound," IEEE Trans. Haptics, vol. 3, no. 3, pp. 155-165, 2010.

[7] H. Lee, J.-S. Kim, S. Choi, J.-H. Jun, J.-R. Park, A.-H. Kim, H.-B. Oh, H.-S. Kim, and S.-C. Chung, "Mid-air tactile stimulation using laserinduced thermoelastic effects: the first study for indirect radiation," in Proc. WHC. IEEE, 2015, pp. 374-380.

[8] Advisory Group on Non-ionising radiation, Health Effects of Exposure to Ultrasound and Infrasound (RCE-14). UK Health Protection Agency, 2010.

[9] G. Ter Haar and C. Coussios, "High intensity focused ultrasound: past, present and future," Int. J. Hyperthermia, vol. 23, no. 2, pp. 85-87, 2007.

[10] L. Gavrilov and E. Tsirulnikov, "Focused ultrasound as a tool to input sensory information to humans (review)," Acoust. Phys+., vol. 58, no. 1, pp. 1-21, 2012.
[11] M. Fink, "Time reversal of ultrasonic fields. I. Basic principles," IEEE Trans. Ultrason. Ferroelectr. Freq. Control., vol. 39, no. 5, pp. 555566, 1992.

[12] M. Fink, C. Prada, F. Wu, and D. Cassereau, "Self focusing in inhomogeneous media with "time reversal" acoustic mirrors," in Proc. IEEE Ultrason. Symp. IEEE, 1989, pp. 681-686.

[13] H. Kajimoto, N. Kawakami, S. Tachi, and M. Inami, "Smarttouch: Electric skin to touch the untouchable," IEEE Comput. Graph. Appl., vol. 24, no. 1, pp. 36-43, 2004.

[14] D. Dalecki, S. Z. Child, C. H. Raeman, and E. L. Carstensen, "Tactile perception of ultrasound," J. Acoust. Soc. Am., vol. 97, no. 5, pp. 3165-3170, 1995.

[15] L. R. Gavrilov, G. V. Gersuni, O. B. Ilyinski, E. M. Tsirulnikov, and E. E. Shchekanov, "A study of reception with the use of focused ultrasound. I. Effects on the skin and deep receptor structures in man," Brain. Res., vol. 135, no. 2, pp. 265-277, 1977.

[16] L. R. Gavrilov and E. M. Tsirulnikov, "Mechanisms of stimulation effects of focused ultrasound on neural structures: Role of nonlinear effects," Nonlinear Acoust. 21st Century, pp. 445-448, 2002.

[17] B. E. Anderson, M. Griffa, C. Larmat, T. J. Ulrich, and P. A. Johnson, "Time reversal," Acoustics Today, vol. 4, no. 1, pp. 5-16, 2008.

[18] M. R. Bai and Y. K. Tsai, "Impact localization combined with haptic feedback for touch panel applications based on the time-reversal approach," J. Acoust. Soc. Am., vol. 129, no. 3, pp. 1297-1305, 2011.

[19] R. K. Ing, N. Quieffin, S. Catheline, and M. Fink, "In solid localization of finger impacts using acoustic time-reversal process," Appl. Phys. Lett., vol. 87, no. 20, p. 204104, 2005.

[20] C. Hudin, J. Lozada, and V. Hayward, "Localized tactile stimulation by time-reversal of flexural waves: Case study with a thin sheet of glass," in Proc. WHC. IEEE, 2013, pp. 67-72.

[21] A. Derode, A. Tourin, and M. Fink, "Ultrasonic pulse compression with one-bit time reversal through multiple scattering," J. Appl. Phys., vol. 85, no. 9, pp. 6343-6352, 1999.

[22] G. Montaldo, P. Roux, A. Derode, C. Negreira, and M. Fink, "Generation of very high pressure pulses with 1-bit time reversal in a solid waveguide," J. Acoust. Soc. Am., vol. 110, no. 6, pp. 2849-2857, 2001.

[23] P. A. Payne, "Measurement of properties and function of skin," Clin. Phys. Physiol. Meas., vol. 12, no. 2, pp. 105-129, 1991.

[24] J. T. Bushberg, J. A. Seibert, E. M. Leidholdt Jr., and J. M. Boone, "The essential physics of medical imaging. Second Edition. 2002," Eur. J. Nucl. Med. Mol. Imaging, vol. 30, p. 1713, 2003.

[25] A. Mujibiya, X. Cao, D. S. Tan, D. Morris, S. N. Patel, and J. Rekimoto, "The sound of touch: On-body touch and gesture sensing based on transdermal ultrasound propagation," in Proc. ACM ITS. ACM, 2013, pp. $189-198$.

[26] D. Dalecki, "Mechanical bioeffects of ultrasound," Annu. Rev. Biomed. Eng., vol. 6, pp. 229-248, 2004.

[27] L. R. Gavrilov, E. M. Tsirulnikov, and I. a. I. Davies, "Application of focused ultrasound for the stimulation of neural structures," Ultrasound Med. Biol., vol. 22, no. 2, pp. 179-192, 1996.

[28] Food and Drug Administration, Information for Manufacturers Seeking Marketing Clearance of Diagnostic Ultrasound Systems and Transducers. U.S. Dept. of Health and Human Services, 1998.

[29] Olympus NDT. (2006) Ultrasonic transducers technical notes. [Online]. Available: https://www.olympus-ims.com/data/File/ panametrics/UT-technotes.en.pdf

[30] I. Poupyrev and S. Maruyama, "Tactile interfaces for small touch screens," in Proc. ACM UIST. ACM, 2003, pp. 217-220.

[31] J. O. Wobbrock, L. Findlater, D. Gergle, and J. J. Higgins, "The aligned rank transform for nonparametric factorial analyses using only ANOVA procedures," in Proc. CHI. ACM, 2011, pp. 143-146. 